\title{
Wheat from the chaff
}

Mathematical modelling is almost as old as science. But the nature of applied mathematics is changing - in physics as well as in biology and medicine, engineering and even economics. The rise of powerful computation has made it possible to create and study models with unprecedented complexity; and to gather equally massive data to use in testing those models.

But a model with 30 or 100 free parameters has enormous flexibility. Can it really ever be tested? Or can a sufficiently clever scientist always manage to adapt it to the data? Science has always worked by putting creative hypotheses and ideas to the experimental test. In the face of highdimensional modelling, could this practice run into trouble?

I've wondered about this question in the context of recent efforts to build models of whole economies. Physicists have been influential in doing so, making significant advances over traditional economic models. But a lingering worry remains - how can you trust a model with a dozen or more variables, and avoid fooling yourself about its explanatory capacity?

Well, physicist Mark Transtrum and colleagues may have found an answer or, at least, they make an encouraging suggestion (M. K. Transtrum et al., preprint at http://arxiv.org/abs/1501.07668; 2015). The problem may not be as vexing as it seems, they argue, because many, if not most, high-dimensional models, as well as real processes, are 'sloppy' - that is, their behaviour depends on very few parameters or details, and the rest are mostly irrelevant. And, they suggest, this isn't just a happy accident; it happens for deep reasons.

Physicists understand well enough how complexity often reduces to simplicity; lots of physics, after all, relies on lowdimensional effective models and theories that work despite ignoring masses of microscopic detail. It's true in statistical physics, thermodynamics or fluid dynamics, and this seeming miracle rests on well understood theory - the continuum limit, or renormalization group arguments. Cartoon theories, Transtrum and colleagues note, can be useful even if they ignore a lot - as long as they get a few crucial things right.

For example, liquids like water or petroleum differ completely at the molecular level, yet on macroscopic

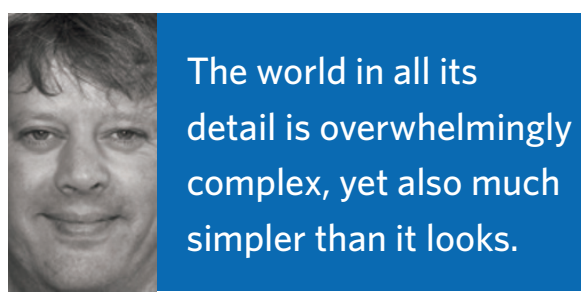

low dimension, retaining only a few basic spatiotemporal modes, captures a large fraction of the variation in the data. The generic sloppiness of natural processes may be a natural explanation.

Sloppiness, the authors argue, may also explain why many biological systems are so robust to environmental variations. The circadian rhythm in cyanobacteria, for example, maintains a 24-hour cycle over a wide range of temperatures, even as chemical reaction rates of the key proteins involved double over this range. This may tell us something about how evolution has exerted control, focusing on a few stiff parameters, yet also benefiting from the low sensitivity of the circuit to other parameters. Engineering such control may not be as difficult as it seems, given the existence and availability of many neutral dimensions for adjustment.

Transtrum and colleagues also argue that sloppiness probably explains why humans - and many other animals - are so good at visual pattern recognition, and reliant on it for interacting with the external world. Pattern recognition means locking on to low-dimensional representations, and ignoring huge volumes of other data. We don't need any further detail, perhaps, because the objects we need to identify - faces, for example - admit lowdimensional representations capturing most of the important information.

Finally, sloppiness might even explain why science itself is possible. The world in all its detail - in anything from ecology to macroeconomics to astrophysics - is overwhelmingly complex, yet also much simpler than it looks. Vastly simplified models can always capture important features, although they of course leave others out (in many systems, for example, influences on single elements truly can lead to macro changes). We do science with lowdimensional models because more complex models are typically less efficient, dwelling on details of marginal importance.

Transtrum and colleagues, it seems, started out thinking about how to analyse data and build models useful for systems biology. Rather than just applying to biology, however, their findings may be much more general. Complexity is a barrier to understanding, but it's not nearly as impenetrable as it seems. analysis often finds that a description of 